\title{
LCMR1 interacts with DEK to suppress apoptosis in lung cancer cells
}

\author{
YANG XU, ZHIXIN LIANG, CHUNSUN LI, ZHEN YANG and LIANGAN CHEN \\ Department of Respiratory Diseases, Chinese PLA General Hospital, Beijing 100853, P.R. China
}

Received September 3, 2016; Accepted May 22, 2017

DOI: $10.3892 / \mathrm{mmr} .2017 .7095$

\begin{abstract}
To win the war against lung cancer, the molecular mechanisms underlying its oncogenesis and metastasis must be identified in order to develop novel diagnosis and treatment strategies. We previously identified a novel gene, namely lung cancer metastasis related protein 1 (LCMR1; GenBank accession no. AY148462), which was demonstrated to be overexpressed in non-small-cell lung cancer. LCMR1 expression was significantly associated with clinical stage. To further understand the mechanism of LCMR1 in lung cancer, the present study screened a cDNA library from the lung cancer cell line 95D for proteins interacting with LCMR1 by yeast two-hybrid assay, and the protein DEK was identified. Co-immunoprecipitation and glutathione S-transferase pull-down assays were performed to confirm the interaction between LCMR1 and DEK in vivo and in vitro. The results demonstrated that the interaction was mediated primarily by the N-terminal region of DEK, suggesting that LCMR1 may be involved in the regulation of cell apoptosis. Using RNA interference, DEK and LCMR1 were demonstrated to cooperate in the inhibition of apoptosis in lung cancer cells, and this effect was associated with the induced myeloid leukemia protein cell differentiation protein 1 pathway. The present findings suggest that LCMR1 might serve as a potential molecular target for lung cancer therapy.
\end{abstract}

\section{Introduction}

Lung cancer is not only the most common cancer worldwide, but also the leading cause of cancer mortality (1). In 2012 alone, 652,842 patients were diagnosed with lung cancer in China, and 597,182 patients died from this disease (2). Worse still, the number of new cases and mortality are increasing dramatically every year (3). To win the war against lung

Correspondence to: Professor Liangan Chen, Department of Respiratory Diseases, Chinese PLA General Hospital, 28 Fuxing Road, Beijing 100853, P.R. China

E-mail: chenla_301@163.com

Key words: lung cancer, lung cancer metastasis related protein 1, protein-protein interaction, DEK proto-oncogene, cell apoptosis cancer, besides advances in current available therapies such as chemotherapy, the molecular mechanisms underlying this disease must be elucidated in order to provide novel targets for targeted therapies (4).

Lung cancer metastasis related protein 1 (LCMR1) is a novel gene cloned for the first time in 2002 in our laboratory from a human large-cell lung carcinoma cell line with high metastaticability, using a differential display polymerase chain reaction technique (5). LCMR1 (GenBank accession no. AY148462) is located on the human chromosome locus 11q12.1, and contains 949 nucleotides with an open reading frame encoding for a peptide with 177 amino acids. In the human genome, LCMR1 is also known as the mediator complex subunit 19 (Med19), which was discovered as a coactivator for DNA-binding factors that activate transcription via RNA polymerase II (6). Recently, Med19 has been reported to serve key roles in promoting numerous types of cancer, including prostate cancer (7), gastric cancer (8), colorectal cancer (9), osteosarcoma (10) and bladder urothelial carcinoma (11). Our previous study reported that LCMR1 is overexpressed in non-small-cell lung cancer (NSCLC) and its expression is significantly associated with the clinical stage (5). In addition, knockdown of LCMR1 in vitro promotes apoptosis of lung cancer cells (12). However, the mechanism underlying its functions remains unclear.

The human DEK proto-oncogene protein (DEK) is a highly conserved nucleoprotein composed of 375 amino acids, which was originally discovered as a fusion with the CAN/NUP214 nucleoporin protein in a subset of acute myeloid leukemia patients carrying the $t(6 ; 9)$ translocation $(13)$. DEK has been reported to be involved in autoimmune disease, viral infection and human carcinogenesis, including melanoma (14), glioblastoma (15), bladder carcinoma (16), and hepatocellular carcinoma (17). Two functional, structured domains of DEK have been identified. The C-terminal domain (amino acids 309-375) includes the region that can reverse the abnormal DNA-mutagen sensitivity of ataxia-telangiectasia cells (18), while the N-terminal domain (amino acids 68-226) confers important in vitro and in vivo functions of DEK, including double-stranded DNA binding, introduction of constrained positive supercoils into closed ds DNA, and apoptosis inhibition (19).

In order to investigate the mechanism of LCMR1 function in the pathogenesis of lung cancer, in the present study, proteins interacting with LCMR1 were screened using a yeast two-hybrid system on a cDNA library from lung cancer 
cell 95D. Protein interaction with LCMR1 was confirmed by co-immunoprecipitation analysis in vivo and glutathione S-transferase (GST) pull-down assay in vitro. The DEK protein was revealed to interact with LCMR1, and the interaction was demonstrated to be primarily mediated by the N-terminal region of DEK. These results suggested that the LCMR1-DEK interaction may be involved in regulation of apoptosis. RNA interference (RNAi) technique was then employed to investigate the effect of DEK-LCMR1 interaction on lung cancer cell apoptosis. The present findings suggest that LCMR1 might serve as a potential target for the development of novel therapies for lung cancer.

\section{Materials and methods}

Cell lines and cell culture. The 95D cell line was a kind gift from Dr Lezhen Chen (Department of Pathology, Chinese PLA General Hospital, Beijing, China). This cell line was generated by subcloning from a poorly-differentiated human large-cell lung carcinoma cell line, PLA-801 (5). 95D cells were cultured in RPMI-1640 (Gibco; Thermo Fisher Scientific, Inc., Waltham, MA, USA) supplemented with $10 \%$ fetal bovine serum (Hyclone; GE Healthcare Life Sciences, Little Chalfont, $\mathrm{UK})$, penicillin $(100 \mu \mathrm{g} / \mathrm{ml})$ and streptomycin $(100 \mu \mathrm{g} / \mathrm{ml})$, at $37^{\circ} \mathrm{C}$ in a humidified $5 \% \mathrm{CO}_{2}$ incubator.

Generation of cDNA library and yeast two-hybrid screening. The total RNA was extracted from 95D cells using TRIzol reagent (Invitrogen; Thermo Fisher Scientific, Inc.). The cDNA library was generated using the BD SMART RACE cDNA Amplification kit (Clontech Laboratories, Inc., Mountain View, CA, USA), according to the manufacturer's instructions. Yeast two-hybrid screening was performed to identify LCMR1-interacting proteins using the Matchmaker GAL4 two-hybrid system III kit (Clontech Laboratories, Inc.), according to the manufacturer's instructions.

GST pull-down assay. GST and GST-LCMR1 fusion proteins were prepared as described previously (20). $\left[{ }^{35} \mathrm{~S}\right]$-methionine-labeled proteins were produced using the TNT Coupled Transcription/Translation system (Promega Corp., Madison, WI, USA), and the expression vectors for DEK (21), and its empty vector control in pcDNA3.0-FLAG. The in vitro transcription/translation reaction products $(20 \mu \mathrm{l})$ were incubated with $100 \mu \mathrm{l}$ GST or GST-LCMR1 fusion proteins bound to glutathione-sepharose beads (Novagen; Merck KGaA, Darmstadt, Germany). The binding reaction was performed for $1 \mathrm{~h}$ at room temperature, and the beads were washed four times, resuspended in $20 \mu 1$ of $2 \mathrm{X}$ SDS-PAGE loading buffer, boiled for $5 \mathrm{~min}$, and resolved on a $12 \%$ SDS-PAGE gel followed by radiophotography.

Co-immunoprecipitation and immunoblotting. A pcDNA3.0-FLAG expression vector directing the expression of DEK (pcDNA3.0-FLAG-DEK) and a pCMV-Myc expression vector directing the expression of LCMR1 (pCMV-Myc-LCMR1) were constructed. 95D cells were seeded in $10 \mathrm{~cm}$ dishes and were transfected at approximately $30-40 \%$ confluency with both $4 \mu \mathrm{g}$ pcDNA3.0-FLAG-DEK and $4 \mu \mathrm{g}$ pCMV-Myc-LCMR1 plasmids together, or either of the plasmid alone using Lipofectamine 2000 (Invitrogen; Thermo Fisher Scientific, Inc.) according to the manufacturer's protocol. At $48 \mathrm{~h}$ post-transfection, cells were harvested and lysed in $500 \mu \mathrm{l}$ lysis buffer containing $20 \mathrm{mM}$ Tris $\mathrm{pH} \mathrm{7.4,}$ $50 \mathrm{mM} \mathrm{NaCl}, 1 \mathrm{mM}$ EDTA, $0.5 \%$ NP-40, 0.5\% SDS, $0.5 \%$ deoxycholate and protease inhibitors. An amount of $500 \mu \mathrm{g}$ of lysate $(1 \mathrm{mg} / \mathrm{ml})$ was precleared with $50 \mu 1$ protein A-Sepharose beads (Upstate Biotechnology, Inc., Lake Placid, NY, USA) for $2 \mathrm{~h}$ at $4^{\circ} \mathrm{C}$. An appropriate amount of rabbit anti-Myc antibody (1:1,000; cat. no. ab32072; Abcam, Cambridge, UK), rabbit anti-FLAG antibody (1:1,000; cat. no. F7425; Sigma-Aldrich, Merck KGaA, Darmstadt, Germany) or rabbit non-specific immunoglobulin G (1:1,000; cat. no. 29070; Clontech Laboratories, Inc.) was then added and incubated overnight at $4^{\circ} \mathrm{C}$. An amount of $100 \mu \mathrm{l}$ of pre-blocked agarose beads was added to the antibody/lysate mixture for another $2 \mathrm{~h}$ at $4^{\circ} \mathrm{C}$, and the beads were pelleted $(0.9 \mathrm{x} \mathrm{g}$ for $1 \mathrm{~min}$ at $4^{\circ} \mathrm{C}$ ) and washed thrice with lysis buffer. Bound proteins were eluted in SDS sample buffer, subjected to $12 \%$ SDS-PAGE and analyzed by immunoblotting. Rabbit anti-Myc (1:1,000; catalog no. ab32072; Abcam), mouse monoclonal anti-FLAG (1:1,000; cat. no. F3040; Sigma-Aldrich, Merck KGaA), and rabbit anti- $\beta$-actin $(1: 150$; cat. no. sc-4778X; Santa Cruz Biotechnology, Inc., Dallas, TX, USA) were used for immunoblotting analyses, as described below.

Small interfering RNA (siRNA) and cell transfection. The human LCMR1-specific siRNA with the sequence 5'-GAGA GAGAGGGACAUGCUU-3', as well as the negative control with the sequence 5'-CCUACGCCACCAAUUUCGU-3', were obtained from Invitrogen to target the LCMR1 sequence (GenBank accession no. AY148462). The siRNA sequence for DEK was 5'-UGUCCUCAUUAAAGAAGAA-3', and the respective negative control sequence was 5'-CUCUAAAGAC AGGUUAUAA-3', also obtained from Invitrogen. 95D cells were seeded in a 6-well plate to reach a $40-50 \%$ confluency, and then $30 \mathrm{nM}$ siRNA was transfected using Lipofectamine 2000 (Invitrogen; Thermo Fisher Scientific, Inc.), according to the manufacturer-recommended protocol.

Reverse transcription-quantitative polymerase chain reaction $(R T-q P C R)$. Total RNA was extracted from the cells using TRIzol reagent (Thermo Fisher Scientific, Inc.) and $2 \mu \mathrm{g}$ RNA was reverse-transcribed using MMLV Reverse Transcriptase (Promega Corp.), according to the manufacturer's protocol. qPCR analysis was performed on a MyiQ2 Real-Time PCR system (Bio-Rad Laboratories, Inc., Hercules, CA, USA), in a volume of $20 \mu \mathrm{l}$ containing $0.5 \mu \mathrm{l}$ cDNA sample, $0.5 \mu 110 \mu \mathrm{M}$ primers, $10 \mu 1$ 2x SYBR Premix Ex Taq (Takara Bio, Inc., Otsu, Japan), and $9 \mu \mathrm{lddH_{2 }}$ O. Cycling conditions were as follows: An initial predenaturation step at $95^{\circ} \mathrm{C}$ for $30 \mathrm{sec}$, followed by 40 cycles of denaturation at $95^{\circ} \mathrm{C}$ for $5 \mathrm{sec}$, annealing at $55^{\circ} \mathrm{C}$ for $15 \mathrm{sec}$, extension at $72^{\circ} \mathrm{C}$ for $15 \mathrm{sec}$ and a final extension at $95^{\circ} \mathrm{C}$ for $1 \mathrm{~min}$. The relative quantity of mRNA was calculated using the $2^{-\Delta \Delta \mathrm{Cq}}$ method (22) with the $\beta$-actin mRNA level as the reference for normalization. All experiments were repeated at least three times. Primer sequences were as follows: $\beta$-actin forward, 5'-CATGTACGTTGCTATCCAGGC-3' and reverse 5'-CTCCTTAATGTCACGCACGAT-3'; LCMR1 forward, 5'-AACAGAGCCGTACCCAGGAT-3' and reverse, 5'-GGG 
A

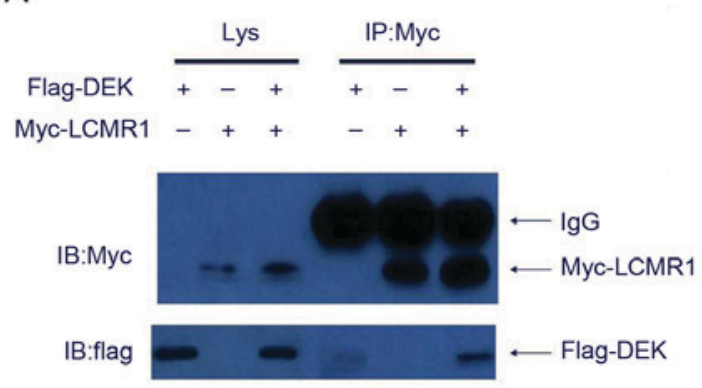

B

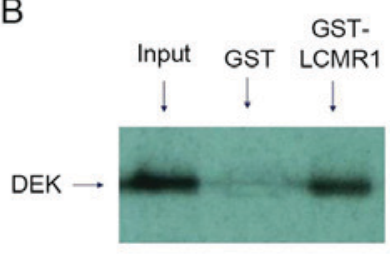

C

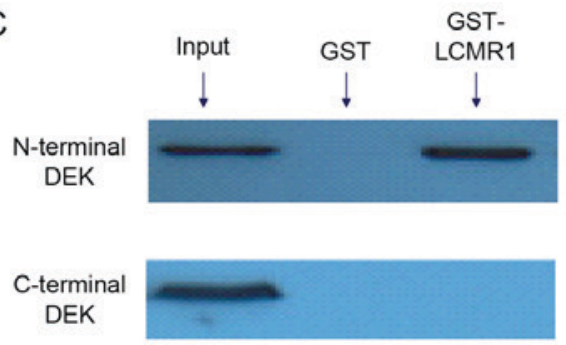

Figure 1. LCMR1 interacts with DEK in vivo and in vitro. (A) The interaction of LCMR1 and DEK in vivo was examined by co-IP assay in 95D lung cancer cells. The proteins identified are indicated by arrows. (B) Direct interaction of LCMR1 and DEK in vitro was examined by GST pull-down assay. (C) $\left[{ }^{35} \mathrm{~S}\right]$-methionine-labeled N-terminal and C-terminal constructs of DEK were prepared and then subjected to GST pull-down assays to examine their interaction with LCMR1. The resulting proteins were resolved on a 12\% SDS-PAGE gel followed by radiophotography. LCMR1, lung cancer metastasis related protein 1; DEK, DEK proto-oncogene; GST, glutathione S-transferase; Lys, lysate; IP, immunoprecipitation.

TGGTCTGGACATTGTC-3'; induced myeloid leukemia protein cell differentiation protein 1 (Mcl-1) forward, 5'-CTC ATTTCTTTTGGTGCCTTT-3' and reverse, 5'-CCAGTCCCG TTTTGTCCTTAC-3'.

Protein extraction and western blotting. The total protein of the cells was extracted for $50 \mathrm{~min}$ at $4^{\circ} \mathrm{C}$ by lysis buffer containing $50 \mathrm{mM}$ Tris pH 7.6, $10 \mathrm{mM}$ EDTA, $150 \mathrm{mM}$ $\mathrm{NaCl}, 0.1 \%$ NP-40, $2 \mathrm{mM}$ dithiothreitol, $1 \mathrm{mM}$ phenylmethylsulfonyl fluoride, $0.7 \mu \mathrm{g} / \mathrm{ml}$ pepstatin A, $10 \mu \mathrm{g} / \mathrm{ml}$ leupeptin, and $1 \mu \mathrm{g} / \mathrm{ml}$ aprotinin. The lysates were then centrifuged at $13.8 \mathrm{x} \mathrm{g}$ for $30 \mathrm{~min}$ at $4^{\circ} \mathrm{C}$. The soluble protein concentrations in lysates were determined using a bicinchoninic acid protein assay kit (Pierce; Thermo Fisher Scientific, Inc.).

For western blot analyses, a total of $25-50 \mu \mathrm{g}$ protein was separated on $12 \%$ SDS-PAGE gel and was transferred ontopolyvinylidene fluoridemembrane. The membrane was blocked in 5\% milk in PBS for $1 \mathrm{~h}$ at room temperature and was then incubated with primary antibodies at $4^{\circ} \mathrm{C}$ overnight. Then, the membrane was washed three times with TBS/Tween-20 (TBST), followed by incubation with a horseradish peroxidase-conjugated secondary antibody at room temperature for $1 \mathrm{~h}$. Following washing with TBST three times, the membrane was then developed using enhanced chemiluminescence (Amersham; GE Healthcare Life Sciences). The staining intensity of the bands was quantitated by densitometry using MultiGauge software version 3.2 (Fuji Pharma Co., Ltd., Tokyo, Japan).

The primary antibodies were: $\beta$-actin (1:500; Santa Cruz Biotechnology, Inc.), LCMR1/MED19 (1:1,000; Abcam), caspase-3 (1:1,1000; New England BioLabs, Inc., Ipswich, MA, USA), caspase-8 (1:1,1000; New England BioLabs, Inc.), caspase-9 (1:1,000; New England BioLabs, Inc.), DEK (1:500; Abcam) and Mcl-1 (1:500; Santa Cruz Biotechnology, Inc.). The secondary antibodies (Santa Cruz Biotechnology, Inc.) were: Anti-goat IgG (1:5,000), anti-rabbit IgG (1:5,000) and anti-mouse $\operatorname{IgG}(1: 6,000)$.

Flow cytometry analysis. Cells were harvested $72 \mathrm{~h}$ post-transfection and fixed with $70 \%$ cold ethanol overnight. The next day, cells were centrifuged at $0.1 \mathrm{x} g$ for $5 \mathrm{~min}$ and resuspended in PBS. Following filtering through a 400 mesh $(38 \mu \mathrm{m})$ membrane, the cells were stained with propidium iodide in the dark at $4^{\circ} \mathrm{C}$ for $30 \mathrm{~min}$, and analyzed using flow cytometry. All experiments were conducted in triplicate.

Statistical analysis. All quantified data represent an average of at least three independent repeats. Data were expressed as the means \pm standard deviation. Differences between groups were analyzed for significance by one-way or two-way analysis of variance following by the Tukey's test, using GraphPad Prism software version 5 (GraphPad Software, Inc., La Jolla, CA, USA). $\mathrm{P}<0.05$ was considered to indicate a statistically significant difference.

\section{Results}

LCMR1 interacts with DEK. A cDNA library, constructed from the 95D lung cancer cell line, was screened for proteins interacting with LCMR1 using a yeast two-hybrid system. Nine clones corresponding to six different potential LCMR1-binding proteins were identified. One such cDNA clone contained the full-length coding sequence for DEK. The specificity of the interaction between LCMR1 and DEK was demonstrated using a chromogenic assay with X-Gal (data not shown).

Co-immunoprecipitation was used to confirm the interaction between LCMR1 and DEK in vivo in 95D cells. The pcDNA3.0-FLAG-DEK plasmid and the pCMV-Myc-LCMR1 plasmid were transfected into 95D cells. Cell lysates were immunoprecipitated with antibodies against the Myc tag. Precipitates were resolved by gel electrophoresis and probed with an antibody against the FLAG tag. An intense band corresponding to Flag-DEK was detected in the anti-Myc antibody immunoprecipitates from the LCMR1 and DEK co-transfection group (Fig. 1A). By contrast, no band was detected in the control groups transfected with any one plasmid alone (Fig. 1A).

GST pull-down was then used to confirm the specificity of the interaction between DEK and LCMR1 in vitro. $\left[{ }^{35} \mathrm{~S}\right]$-methionine-labeled full-length DEK was incubated with GST-LCMR1 or GST alone. The results demonstrated that the 

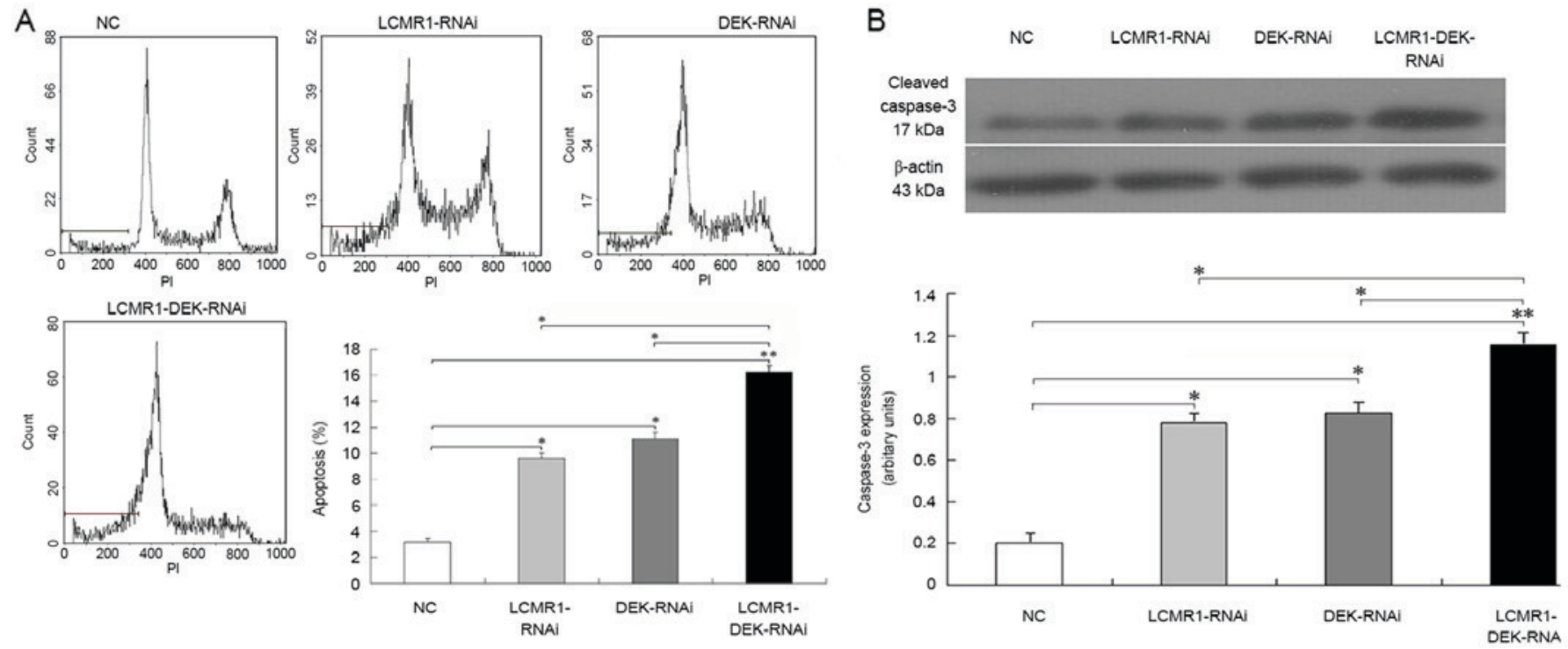

Figure 2. DEK and LCMR1 cooperate to suppress the apoptosis of lung cancer cells. (A) Apoptosis of 95D cells was analyzed by flow cytometry at $72 \mathrm{~h}$ post-transfection with small interfering RNA for LCMR1, DEK or both. The graph demonstrates comparisons of the numbers of apoptotic cells between the control (NC) group, the small interfering RNA for LCMR1, DEK or both, as well as comparisons of the numbers of apoptotic cells between the double knockdown group and the single knockdowns. (B) Protein expression levels of cleaved caspase- 3 were examined by western blotting. $\beta$-actin was used as internal control. The graph demonstrates comparisons of the protein expression levels of cleaved caspase-3 between the control (NC) group and small interfering RNA for LCMR1, DEK or both, as well as comparisons of the protein expression levels of cleaved caspase-3 between the double knockdown group and the single knockdowns. Data are presented as mean \pm standard deviation. " $\mathrm{P}<0.05$ and ${ }^{* *} \mathrm{P}<0.01$. DEK, DEK proto-oncogene; LCMR1, lung cancer metastasis related protein 1; RNAi, RNA interference.

full-length DEK polypeptide bound strongly to GST-LCMR1 but failed to bind to GST alone (Fig. 1B). Together, these data demonstrate a direct physical interaction between LCMR1 and DEK in vitro and in vivo.

Two structured domains of DEK have been reported to be associated with different functions. To explore the potential functional implications of the DEK-LCMR1 interaction, N-terminal and C-terminal deletion constructs of DEK were generated and used in GST pull-down assays, in an effort to identify the region of DEK that is required for LCMR1 binding. As demonstrated in Fig. 1C, GST-LCMR1 bound strongly to the N-terminal DEK construct (amino acids 68-226), but failed to bind to the C-terminal DEK construct (amino acids 309-375). These results revealed that the interaction between DEK and LCMR1 is primarily mediated by the $\mathrm{N}$-terminal region of DEK.

DEK and LCMRI cooperate to suppress apoptosis of lung cancer cells. It has been reported that the N-terminal region of the DEK protein is implicated in apoptosis regulation. To determine the effect of the interaction of LCMR1 and DEK on apoptosis, apoptosis rates were examined by flow cytometry in 95D cells following siRNA knockdown of LCMR1 (LCMR1-RNAi), DEK (DEK-RNAi) or both (LCMR1-DEK-RNAi). As illustrated in Fig. 2A, the \% of apoptotic cells in the LCMR1-RNAi and DEK-RNAi groups was significantly higher compared with the control group $(\mathrm{P}<0.05)$. In addition, the double knockdown LCMR1-DEK-RNAi group exhibited the highest percentage of apoptotic cells $(\mathrm{P}<0.01)$. As compared with the single knockdowns alone, the double knockdown exhibited a significantly greater percentage of apoptotic cells (Fig. 2A; $\mathrm{P}<0.05$ ). These results demonstrate that knockdown of either LCMR1 or DEK by siRNA, as well as the double knockdown, promoted apoptosis in 95D cells.
To further confirm the effect of the interaction of LCMR1 and DEK in apoptosis, the expression levels of cleaved caspase-3 were compared among the LCMR1-RNAi, DEK-RNAi, and LCMR1-DEK-RNAi groups using western blot analysis. Consistent with the aforementioned flow cytometry results, the expression levels of cleaved caspase-3 protein in the LCMR1-RNAi and the DEK-RNAi groups were significantly higher than the control group, and the double knockdown LCMR1-DEK-RNAi group exhibited the highest levels $(\mathrm{P}<0.01)$. As compared with the single knockdowns, the double knockdown exhibited significantly higher expression levels of cleaved caspase-3 protein (Fig. $2 \mathrm{~B}, \mathrm{P}<0.05$ ). Taken together, these findings suggest that DEK and LCMR1 cooperate to suppress the apoptosis of lung cancer cells.

LCMR1 knockdown induces apoptosis through both the caspase- 8 and caspase-9 pathways. To further confirm the mechanism of LCMR1-related apoptosis, the expression levels of cleaved caspase-8 (a marker of death receptor apoptotic pathways) and cleaved caspase-9 (a marker of mitochondrial apoptotic pathways) were examined in LCMR1-RNAi cells using western blot analysis. As demonstrated in Fig. 3, the expression levels of both cleaved caspase- 8 protein (Fig. 3A) and cleaved caspase- 9 protein (Fig. 3B) in the LCMR1-RNAi group were significantly higher compared with the untreated and control-transfected groups. These findings suggest that LCMR1 knockdown potentiated apoptotic activity in 95D cells through both the death receptor and the mitochondrial apoptotic pathways.

LCMR1/DEK-related apoptosis is associated with Mcl-1. It has been reported that DEK-mediated apoptosis is associated with Mcl-1 activity (23). To further explore the mechanism of LCMR1 and DEK-related apoptosis, the expression levels of Mcl-1 in 
A
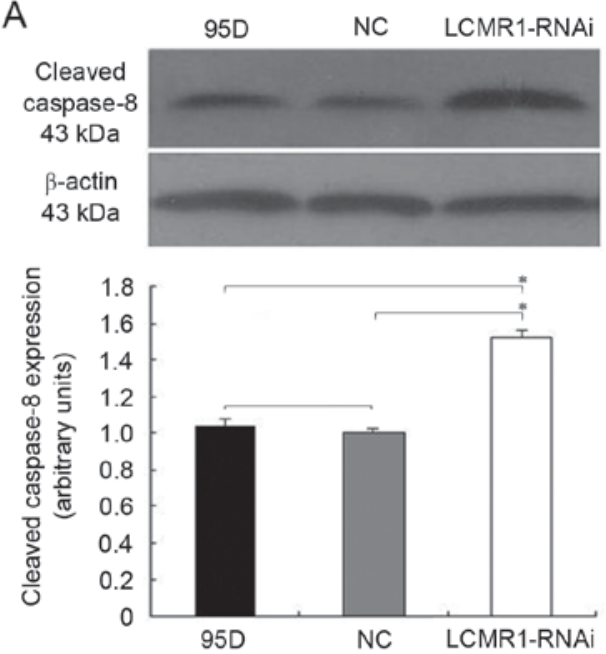

B
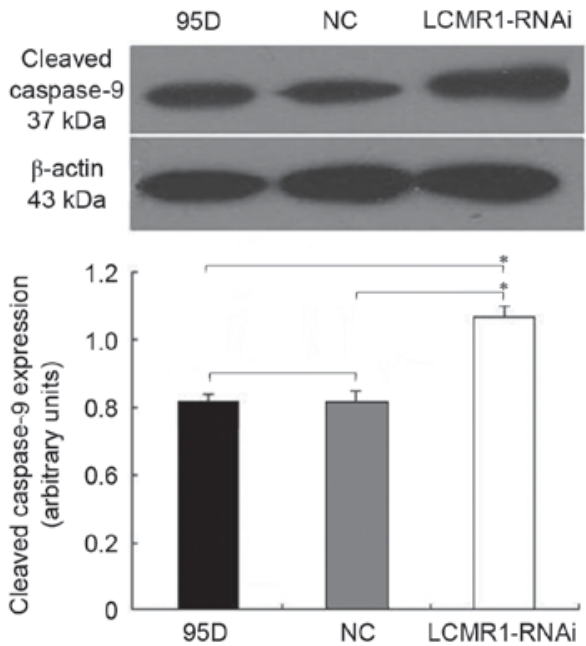

Figure 3. LCMR1 knockdown induces apoptosis through both caspase-8 and caspase-9 pathways. Protein expression levels of (A) cleaved caspase-8 and (B) cleaved caspase-9 were examined by western blot analysis in untreated 95D cells (95D) and cells transfected with either negative control siRNA (NC), or LCMR1-specific siRNA (LIMCR1-RNAi). $\beta$-actin was used as internal control. Data are presented as mean \pm standard deviation. * $<0.05$ compared with control (NC) group and untreated 95D cells (95D). LCMR1, lung cancer metastasis related protein 1; siRNA, small interfering RNA; RNAi, RNA interference.

the LCMR1-RNAi, DEK-RNAi, and LCMR1-DEK-RNAi groups were examined by RT-qPCR and western blotting. The results demonstrated that Mcl-1 expression was significantly downregulated at both the mRNA level (Fig. 4A) and the protein level (Fig. 4B) in the LCMR1-RNAi and DEK-RNAi groups compared with the control group $(\mathrm{P}<0.05)$, while those in the LCMR1-DEK-RNAi group decreased even further. We compared the Mcl-1 expression at both the mRNA level and the protein level between the double knockdown group and the single knockdown groups, and results revealed that the double knockdown group was significantly downregulated at both the mRNA level (Fig. 4A; P<0.05) and the protein level (Fig. 4B; $\mathrm{P}<0.05)$. These results suggest that LCMR1 and DEK-mediated apoptosis is associated with the Mcl-1 pathway.

\section{Discussion}

In order to investigate the function of LCMR1 in the pathogenesis of lung cancer, the present study screened for proteins interacting with LCMR1 using a yeast two-hybrid system, and then confirmed the specific interaction with LCMR1 by co-immunoprecipitation analysis in vivo in 95D cells and GST pull-down assay in vitro. The results demonstrated that the DEK protein specifically interacted with LCMR1.

DEK has an established association with carcinogenesis, and the DEK gene was originally identified in the $t(6 ; 9)$ chromosomal translocation in a subtype of patients with acute myelogenous leukemia (13). Later reports have associated DEK with various diseases, ranging from cancer to autoimmune diseases $(15-17,24)$. The 375 amino acid human protein DEK contains two functional structured domains, namely the N-terminal domain (amino acids 68-226) and the C-terminal domain (amino acids 309-375) (18,19). In the present study, GST-LCMR1 failed to bind to C-terminal DEK but bound strongly to $\mathrm{N}$-terminal DEK, indicating that the interaction between DEK and LCMR1 is mediated primarily by the $\mathrm{N}$-terminal region of DEK. The N-terminal region of DEK has been reported to confer important apoptosis inhibition
A

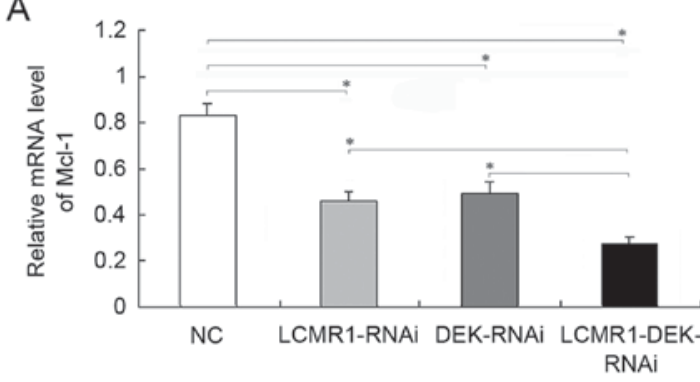

B
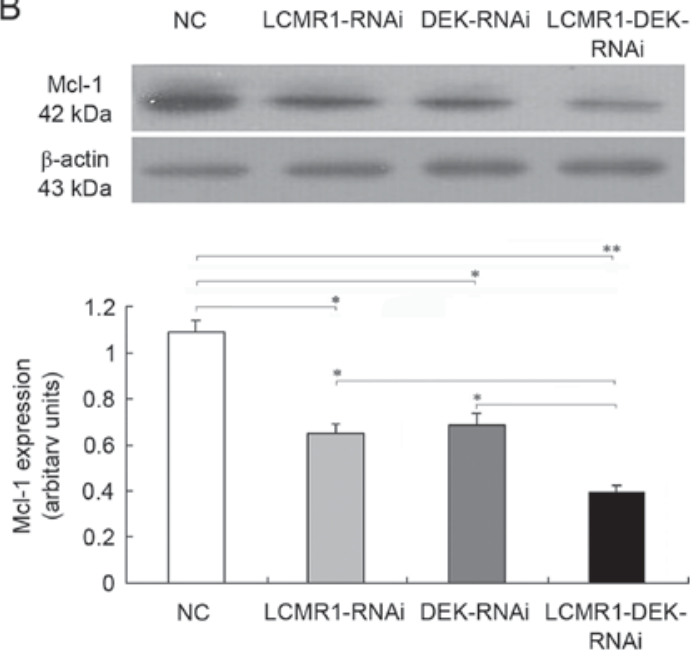

Figure 4. LCMR1 and DEK-related apoptosis are associated with Mcl-1. The expression of Mcl-1 was examined by (A) reverse transcription-quantitative polymerase chain reaction and (B) western blotting in 95D cells transfected with small interfering RNA for LCMR1, DEK or both. $\beta$-actin was used as internal control in western blot analyses. Data are presented as mean \pm standard deviation. ${ }^{*} \mathrm{P}<0.05$ and ${ }^{* *} \mathrm{P}<0.01$. LCMR1, lung cancer metastasis related protein 1; DEK, DEK proto-oncogene; Mcl-1, induced myeloid leukemia protein cell differentiation protein 1; RNAi, RNA interference.

functions, which provided essential clues for the investigation of the LCMR1-DEK interaction functions. 
To determine the effect of the interaction of LCMR1 and DEK in the apoptotic activity in 95D cells, apoptosis was examined by flow cytometry and caspase-3 expression following siRNA knockdown. The results demonstrated that DEK and LCMR1 cooperated to suppress apoptosis of lung cancer cells. It has been reported that the proto-oncogene protein DEK induces apoptotic cell death through caspase-9 and caspase-3-dependent pathways (25). The present study explored the expression levels of cleaved caspase-8 and cleaved caspase-9 following LCMR1 knockdown, and demonstrated that both the death receptor and the mitochondrial apoptotic pathways were involved. These findings imply that LCMR1 may interact with apoptosis-related proteins other than DEK. Further studies are underway to reveal novel potential LCMR1-binding proteins and their mechanisms.

Mcl-1 is an antiapoptotic protein of the Bcl-2 family, which is highly expressed in various types of cancer and results in evading cell death and developing drug resistance, including in breast, colon, lung, renal and prostate cancer (26). Mcl-1 is regulated at both the transcriptional and translational levels. Transcriptionally, Mcl-1 expression can be induced by a variety of cytokines and signaling pathways, including the phosphoinositide 3-kinase/AKT, signal transducer and activator of transcription 3 , and p38/mitogen activated protein kinase pathways (27). At the translational level, several microRNAs have been reported to have important roles in Mcl-1 regulation (28). Previously, it has been revealed that DEK-mediated apoptosis is associated with Mcl-1 (23). Therefore, in the present study, the expression levels of Mcl-1 were examined following LCMR1 and/or DEK knockdown. The results demonstrated a dramatic decrease in Mcl-1 expression in the knockdown groups compared with control, which suggested that LCMR1 and/or DEK-mediated apoptosis are associated with the Mcl-1 pathway. Further studies will be required to explore the specific mechanism.

In conclusion, the present study identified DEK as a novel interacting protein for LCMR1 and demonstrated that the interaction was mediated through the $\mathrm{N}$-terminal region of DEK. Using RNAi, it was revealed that DEK and LCMR1 cooperated to suppress apoptosis in lung cancer cells, and this effect may be associated with the Mcl-1 pathway. The present findings further suggest that LCMR1 might serve as a potential molecular target for lung cancer therapy.

\section{References}

1. Siegel RL, Miller KD and Jemal A: Cancer statistics, 2015. CA Cancer J Clin 65: 5-29, 2015.

2. Shi Y and Sun Y: Medical management of lung cancer: Experience in China. Thorac Cancer 6: 10-16, 2015.

3. Chen W, Zheng R, Zeng $\mathrm{H}$ and Zhang S: Epidemiology of lung cancer in China. Thorac Cancer 6: 209-215, 2015.

4. Rothschild SI: Targeted therapies in non-small cell lung cancer-beyond EGFR and ALK. Cancers 7: 930-949, 2015.

5. Chen L, Liang Z, Tian Q, Li C, Ma X, Zhang Y, Yang Z, Wang P and Li Y: Overexpression of LCMR1 is significantly associated with clinical stage in human NSCLC. J Exp Clin Cancer Res 30: $18,2011$.

6. Sato S, Tomomori-Sato C, Parmely TJ, Florens L, Zybailov B, Swanson SK, Banks CA, Jin J, Cai Y, Washburn MP, et al: A set of consensus mammalian mediator subunits identified by multidimensional protein identification technology. Mol Cell 14: 685-691, 2004

7. Cui X, Xu D, Lv C, Qu F, He J, Chen M, Liu Y, Gao Y, Che J, Yao Y and Yu H: Suppression of MED19 expression by shRNA induces inhibition of cell proliferation and tumorigenesis in human prostate cancer cells. BMB reports 44: 547-552, 2011.
8. Ding XF, Huang GM, Shi Y, Li JA and Fang XD: Med19 promotes gastric cancer progression and cellular growth. Gene 504: 262-267, 2012.

9. Ji-Fu E, Xing JJ, Hao LQ and Fu CG: Suppression of lung cancer metastasis-related protein 1 (LCMR1) inhibits the growth of colorectal cancer cells. Mol Biol Rep 39: 3675-3681, 2012.

10. Yu W, Zhang Z, Min D, Yang Q, Du X, Tang L, Lin F, Sun Y, Zhao H, Zheng S, et al: Mediator of RNA polymerase II transcription subunit 19 promotes osteosarcoma growth and metastasis and associates with prognosis. Eur J Cancer 50: 1125-1136, 2014.

11. Wen H, Feng CC, Ding GX, Meng DL, Ding Q, Fang ZJ, Xia GW, $\mathrm{Xu}$ G and Jiang HW: Med19 promotes bone metastasis and invasiveness of bladder urothelial carcinoma via bone morphogenetic protein 2. Ann Diagn Pathol 17: 259-264, 2013.

12. Xu Y,Li C, Tian Q, Li Y, Yang Z,Liang Z and Chen L: Suppression of lung cancer metastasis-related protein 1 promotes apoptosis in lung cancer cells. Int J Mol Med 30: 1481-1486, 2012.

13. von Lindern M, Fornerod M, van Baal S, Jaegle M, de Wit T, Buijs A and Grosveld G: The translocation (6;9), associated with a specific subtype of acute myeloid leukemia, results in the fusion of two genes, dek and can, and the expression of a chimeric, leukemia-specific dek-can mRNA. Mol Cell Biol 12: 1687-1697, 1992.

14. Grottke C, Mantwill K, Dietel M, Schadendorf D and Lage H: Identification of differentially expressed genes in human melanoma cells with acquired resistance to various antineoplastic drugs. Int J Cancer 88: 535-546, 2000.

15. Kroes RA, Jastrow A, McLone MG, Yamamoto H, Colley P, Kersey DS, Yong VW, Mkrdichian E, Cerullo L, Leestma J and Moskal JR: The identification of novel therapeutic targets for the treatment of malignant brain tumors. Cancer Lett 156: 191-198, 2000.

16. Evans AJ, Gallie BL, Jewett MA, Pond GR, Vandezande K, Underwood J, Fradet Y, Lim G, Marrano P, Zielenska M and Squire JA: Defining a $0.5-\mathrm{mb}$ region of genomic gain on chromosome 6 p22 in bladder cancer by quantitative-multiplex polymerase chain reaction. Am J Pathol 164: 285-293, 2004.

17. Kondoh N, Wakatsuki T, Ryo A, Hada A, Aihara T, Horiuchi S, Goseki N, Matsubara O, Takenaka K, Shichita M, et al: Identification and characterization of genes associated with human hepatocellular carcinogenesis. Cancer Res 59: 4990-4996, 1999.

18. Devany M, Kotharu NP and Matsuo H: Structure of the C-terminal domain of the human protein DEK. Protein Sci 13: 2252-2259, 2004.

19. Devany M, Kappes F, Chen KM, Markovitz DM and Matsuo H: Solution NMR structure of the N-terminal domain of the human DEK protein. Protein Sci 17: 205-215, 2008.

20. Yang J, Zhao YL, Wu ZQ, Si YL, Meng YG, Fu XB, Mu YM and Han WD: The single-macro domain protein LRP16 is an essential cofactor of androgen receptor. Endocr Relat Cance 16: 139-153, 2009.

21. Xu Y,Li C, Tian Q, Li Y, Yang Z,Liang Z and Chen L: Suppression of lung cancer metastasis-related protein 1 promotes apoptosis in lung cancer cells. Int J Mol Med 30: 1481-1486, 2012.

22. Livak KJ and Schmittgen TD: Analysis of relative gene expression data using real-time quantitative PCR and the 2(-Delta Delta C(T)) method. Method 25: 402-408, 2001.

23. Khodadoust MS, Verhaegen M, Kappes F, Riveiro-Falkenbach E, Cigudosa JC, Kim DS, Chinnaiyan AM, Markovitz DM and Soengas MS: Melanoma proliferation and chemoresistance controlled by the DEK oncogene. Cancer Res 69: 6405-6413,2009.

24. Kim DW, Chae JI, Kim JY, Pak JH, Koo DB, Bahk YY and Seo SB: Proteomic analysis of apoptosis related proteins regulated by proto-oncogene protein DEK. J Cell Biochem 106: 1048-1059, 2009

25. Lin L, Piao J, Ma Y, Jin T, Quan C, Kong J, Li Y and Lin Z: Mechanisms underlying cancer growth and apoptosis by DEK overexpression in colorectal cancer. PLoS One 9: e111260, 2014.

26. Quinn BA, Dash R, Azab B, Sarkar S, Das SK, Kumar S, Oyesanya RA, Dasgupta S, Dent P, Grant S, et al: Targeting Mcl-1 for the therapy of cancer. Expert Opin Investig Drugs 20: 1397-1411, 2011.

27. Perciavalle RM and Opferman JT: Delving deeper: CL-1's contributions to normal and cancer biology. Trends Cell Biol 23: 22-29, 2013.

28. Thomas LW, Lam C and Edwards SW: Mcl-1; the molecular regulation of protein function. FEBS Lett 584: 2981-2989, 2010. 